\title{
COMPARING SOME PROPERTIES OF LEFTIST AND RIGHTIST MEASURES OF INEQUALITY
}

\author{
KATARZYNA OSTASIEWICZ \\ Wrocław University of Economics, Faculty of Management, Computer Science and Finance, \\ Department of Statistics, Komandorska 118/120, 53-345 Wrocław, Poland \\ email: katarzyna.ostasiewicz@ue.wroc.pl
}

\begin{abstract}
Most of commonly used measures of inequality belongs to the so-called rightist measures which are defined by scale-invariance. However, there exist also so-called leftist measures, defined by translation invariance. According to surveys of subjective feelings of people, some feel that after multiplying each value of the distribution by the same constant value the inequality remains constant, while the other group feel that the inequality does not change after adding the same value to each value of the distribution. This ambiguity led to proposing by some authors measures of inequality that have properties of both rightist and leftist measures, that is, are invariant over rescaling and over translation. In this paper we examine the potential differences between iso-inequality contour plots for these two groups of measures, confronting with the properties of measure belonging mutually to both of the groups.
\end{abstract}

Key words: rightist inequality measure, leftist inequality measure, iso-inequality contour, inequality measurement

JEL Codes: 1320

DOI: 10.15611/amse.2017.20.27

\section{Introduction}

There are plenty of different existing inequality measures, most of them based on some underlying utility function/social welfare function (e.g. Kot, 2012; Cowell, 2011). Sometimes their relative advantages/disadvantages are compared (e.g. Greseklin et al., 2010; Ostasiewicz, 2014), still, these most commonly used are characterized by scale invariance (e.g. Allison, 1978). That is, inequalities of distribution $\mathcal{B}$ and distribution $\widetilde{\mathcal{B}}$, obtained by multiplying each value of $\mathcal{B}$ by nonzero constant, are exactly the same. However, such property has been questioned (Kolm, 1976a; Kolm 1976b; Kelley, Klein, 1977). Kolm (1976a) recalls the observation of subjective feelings of members of French workers. In 1968 after the general strike Grenelle agreements increased by $13 \%$ all payments - and many of the lower levels of the ladder felt cheated, as according to their feelings inequalities increased. That led to proposing completely new family of inequality measures, so-called "leftist" measures, which are instead of scale-invariance characterized by translation-invariance, that is, adding some constant to each value of distribution $\mathcal{B}$ does not change the value of such a measure of inequality. Scale-invariance measures are thus called "rightist" measures.

The ambiguity of these two possible properties led to proposing by some authors intermediate and compromise measures of inequality (e.g. Kolm, 1999), which are in general neither rightist nor leftist, with rightist and leftist properties as limiting cases. Less attention has been paid to possible measures which are both rightist and leftist. One of few proposition, 
in a particular simple functional form, was the formula given by Gehrig (1980). Independently on the potential perception of this measure of inequality, it seems beneficial to investigate its properties as possibly shedding more light on similarities and differences between leftist and rightist measures.

The paper is organized as follows. In the following section we define the measures taken into regard. Section 3 examines the potential differences between the two groups of measures with regard to curves of inequality plots in the simplest case of two-individulas sharing the resource. Section 4 extends the analysis for another dimension, while the last one offers the summary and conclusions.

All numerical calculations and figures has been prepared by Author with the use of program Mathematica.

\section{Definitions of Rightist and Leftist Measures}

Virtually all most commonly used inequality measures are characterized by scale invariance. That is, if we have distribution $\mathcal{B}$ and transformed distribution $\widetilde{\mathcal{B}}$ which is obtained from $\mathcal{B}$ by:

with $c>0$,

$$
\widetilde{\mathcal{B}}=c B,
$$

then:

$$
I_{R}(\mathcal{B})=I_{R}(\widetilde{\mathcal{B}})
$$

what defines rightist inequality measures, indicated by subscript $R\left(I_{R}\right)$.

Scale invariance seems to be very intuitive property, as inequality measure should not change under changing units in which the values of distribution are measured. E.g., inequality should be the same regardless of whether the income is given in PLN or in thousands of PLN. Scale invariance ensures easygoing comparisons of inequalities between countries and in time. One can compare even inequalities between two completely different resources, e.g. may state a question what is distributed more evenly: incomes or duration of life. Such property is advantageous in many ways, similarly to those for which it is common to construct dimensionless coefficient of variability instead of standard deviation or coefficient of correlation instead of covariance.

Amongst rightist measures of inequality probably the most popular are Gini index, Atkinson family of indices and Theil $\mathrm{T}$ index, and they are chosen to be examined here in this paper. Let distribution $\mathcal{B}$ consists from $n$ values: $\mathcal{B}=\left\{x_{1}, \ldots, x_{n}\right\}$, with average value equal to $\bar{x}$. Then we have the following definitions.

Gini index is defined as (Gini, 1921):

$$
G=\frac{\sum_{i, j}\left|x_{i}-x_{j}\right|}{2 n^{2} \bar{x}} \equiv \frac{\Delta}{2 \bar{x}}
$$

Atkinson family of indices is defined as (Atkinson, 1970):

$$
A=1-\left(\frac{1}{n} \sum_{i}\left(\frac{x_{i}}{\bar{x}}\right)^{1-\epsilon}\right)^{1 /(1-\epsilon)}
$$

where $\epsilon \geq 0$ is called an inequality aversion; and Theil T index as (Theil, 1967):

$$
T=\frac{1}{n} \sum_{i} \frac{x_{i}}{\bar{x}} \ln \frac{x_{i}}{\bar{x}}
$$

It may be easily checked that over transformation: $\left\{x_{i}\right\} \rightarrow\left\{c x_{i}\right\} \quad(c>0)$ each of these measures do not change its value.

However, plausibility of this approach may seem deceptive (see e.g. Amiel, Cowell, 1999; Kjellsson, Gerdtham, 2013). 
Justifying another approach introduced in his paper, Kolm (1976) mentioned the case of French trade unionists, who felt that equiproportional increases in salaries increased inequalities within the workers of their branch of industry. Also, some investigations of subjective perceptions of inequalities show, that under multiplying each value of distribution by a constant larger than one, the inequalities are perceived as increasing (e.g. Harrison, Seidl, 1994).

Thus, the competing property of inequality measures has been proposed. Invariance under translation defines the leftist measures of inequality. The measure of inequality is called leftist (and will be denoted by $I_{L}$ ) if for:

where $I$ denotes distribution $\{1,1, \ldots, 1\}$,

$$
\widetilde{\widetilde{B}}=\mathcal{B}+c I,
$$

it holds:

$$
I_{L}(\mathcal{B})=I_{L}(\widetilde{\widetilde{B}}) .
$$

Virtually the only one measure belonging to this family of indices and generally recognized in the literature is Kolm index (Kolm, 1976a; Kolm, 1976b), defined as:

where $\alpha \geq 0$.

$$
K=\frac{1}{\alpha} \ln \left[\frac{1}{n} \sum_{i=1}^{n} \exp \left[\alpha\left(\bar{x}-x_{i}\right)\right]\right],
$$

Worth noticing is existing of a measure proposed by Gehrig (1980) which is simultaneously both rightist and leftist, that is,

$$
I_{R L}(\mathcal{B})=I_{R L}(\widetilde{\mathcal{B}})=I_{R L}(\widetilde{\mathcal{B}}) .
$$

This measure takes the form:

$$
I_{R L}=\left\{\begin{array}{cc}
\Phi\left(\frac{x_{1}-\min \left\{x_{1}, \ldots, x_{n}\right\}}{x_{1}+\ldots+x_{n}-n \cdot \min \left\{x_{1}, \ldots, x_{n}\right\}}, \ldots, \frac{x_{n}-\min \left\{x_{1}, \ldots, x_{n}\right\}}{x_{1}+\ldots+x_{n}-n \cdot \min \left\{x_{1}, \ldots, x_{n}\right\}}\right) & \text { if not } \quad x_{1}=x_{2}=\ldots=x_{n} \\
0 & \text { if } \quad x_{1}=x_{2}=\ldots=x_{n}
\end{array}\right.
$$

where $\Phi$ denotes any symmetrical function.

Here, we will choose $\Phi$ as power mean:

$$
\Phi\left(z_{1}, \ldots, z_{m}\right)=\left[\frac{1}{m} \sum_{i=1}^{m} z_{i}\right]^{1 / k}
$$

with $k=2$ (with this choice the inequality measure will be denoted by $I_{R L 2}$ ) and $k=3$ (with this choice the inequality measure will be denoted by $\left.I_{R L 3}\right)$.

The maximum value of each of these measures, given the total amount of resource, is met in the case of $x_{1}=x_{2}=\ldots=x_{n-1}=0, x_{n}=n \bar{x}$. What follows, the maximum value of Gini index, $G_{\max }$, equals to:

$$
G_{\max }=\frac{n-1}{n}
$$

the maximum of Atkinson index equals to:

the maximum of Theil index:

$$
A_{\max }=1-\left(\frac{1}{n}\right)^{\epsilon /(1-\epsilon)}
$$

the maximum of Kolm measure:

$$
T_{\max }=\ln n,
$$

$$
K_{\max }=\frac{1}{\alpha} \ln \left[\frac{1}{n}((n-1) \exp [\alpha \bar{x}]+\exp [-\alpha \bar{x}(n-1)])\right],
$$

whilst the maximum of rightist-leftist measure:

$$
I_{\mathrm{RLmax}}=\left(\frac{1}{n}\right)^{1 / k}
$$




\section{Comparison of the Measures: the Case of $n=2$}

In this section we will deal with the simplest case of sharing a total resource by two individuals. Instead of asking a question: what is the value of a given measure of inequality while the share of one person is $x$ (what follows: the share of the second person is $1-x$ ) we will state a reverse question: what should be the share of one of the individuals to reach a given level of inequality?

Let the total resource equals to 1 . Using expressions (12)-(16) with $n=2$ we can plot the share of one of the individuals to get a given value of inequality measure, see Figure 1 for plots for Gini index, Atkinson index with $\epsilon=0.5$, Theil index, Kolm index with $\alpha=0.5$ and $I_{\mathrm{RL}}$ with $k=2$. Each plot is characterized by two branches: of course, due to anonymity, the same level of inequality will be generated with the shares $(x, 1-x)$ and $(1-x, x)$.

Figure 1: Values of measures of inequality versus the share of one of the individual in twoindividuals case

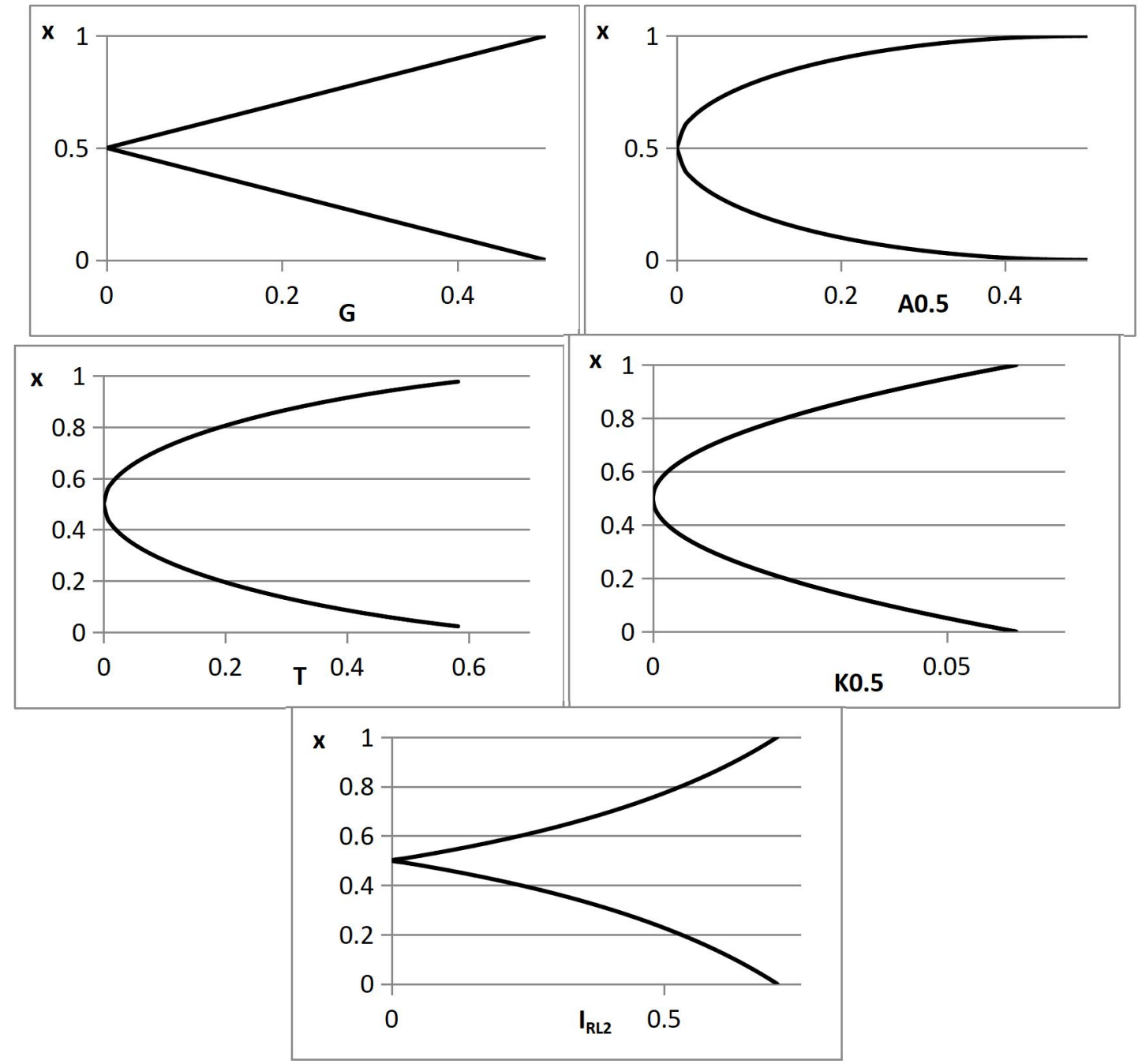

Source: The Author's work

A surprising observation can be made. There are no qualitative differences between Atkinson, Theil and Kolm measures (that is, representatives of rightist and leftist measures), whilst there are such differences between Gini index and all the others - that is, however, a common knowledge of a unique properties of Gini index - but there are also qualitative differences between the group of rightist and leftist measures and the one that possesses the 
properties both rightist and leftist. All the measures with specified "laterality" (rightist/leftist), apart from Gini index, have the concavity just the opposite to the concavity of the curve for both leftist and rightist measure.

To investigate the effect more deeply let us proceed to the case of $n=3$.

\section{Comparison of the Measures: the Case of $n=3$}

For three individuals sharing the resource the plots of the inequality measures becomes three-dimensional. Let us start from comparing two-dimensional iso-inequality contours, which are projections on the plane $I=0$ intersections of $I(x, y, z(x, y))$ and $I=C$, where $I$ denotes a given inequality measure, $x$ is the value of what holds one of the individuals, $y$ the value of what holds the other individual, and $z$ - what holds the third individual. If the total amount of goods is equal to $T$, then: $z(x, y)=T-x-y$. Such contours have been already investigated by Cowell (1985), who found the general form for some subclass of rightist measures.

Figures 2 and 3 present contours for Gini index, Atkinson, Theil and Kolm measures. Again, the striking feature is no evident differences between rightist and leftists measures. The contours for Theil and Atkinson indices are both more resembling those for Kolm measure than for Gini index.

As there are no qualitative differences between rightist and leftist contours, one may expect that those related to $I_{\mathrm{RL}}$ would be similar. However, the results are surprising, as the contours for measure that is both rightist and leftist are topologically different from both rightists contours and leftists ones, see Figure 4.

The key to that strange effect lies probably not in operations to which the particular measures are invariant, but in these ones, under which the given measures undergo changes.

To throw light on the issue let us investigate the case when the total amount of resource to be divided among three persons equals to 2 , that is, $T=2$. Obviously, the zero inequality for all measures shift now from $\left(\frac{1}{3} ; \frac{1}{3} ; \frac{1}{3}\right)$ to the point $\left(\frac{2}{3} ; \frac{2}{3} ; \frac{2}{3}\right)$. However, measures that are scale invariant, for the same value of inequality have to give the contour twice as large as in case $T=1$, while measures that are translation invariant have to give exactly the same contour (the same size) as in the case of $T=1$, cf. plots for Atkinson index and Kolm index, Figure 5.

In the light of this observation it is obvious, that the contours for measure which is both scale-invariant and translation-invariant have to be in the form of straight lines. Straight lines are the only shapes that remains the same after multiplying, and this is a requirement for $I_{\mathrm{RL}}$ measure to both remain the same size (as leftist measures) and be multiplied by the constant (as rightist measures).

Still, it might be interesting, what is the shape of $\mathrm{I}(\mathrm{x}, \mathrm{y}, \mathrm{T}-\mathrm{x}-\mathrm{y})$ that an intersection with the plane gives the straight lines. We have seen (Figure 1) that for two-individuals case the plots of inequality (as functions of share of one of the individuals) have different concavity for both rightist and leftist measures on the one hand and $\mathrm{I}_{\mathrm{RL}}$ measure on the other hand. Here we have seen that the intersections with the plane again for both rightist and leftist measures on the one hand and $\mathrm{I}_{\mathrm{RL}}$ measure on the other hand generate curves topologically different. Let us compare thus three dimensional plots of Gini index, Atkinson index, Theil index, Kolm index, and $\mathrm{I}_{\mathrm{RL}}$ measure, see Figure 6 below.

Indeed, the shape of $\mathrm{I}_{\mathrm{RL}}$ measure again reveals quite interesting form, not being smoothly convex in the whole range of the domain, like all the remaining plots. 
Figure 2: Contour plots of iso-inequality for chosen measures of inequality, Gini index and Atkinson index with $\epsilon=0.5$
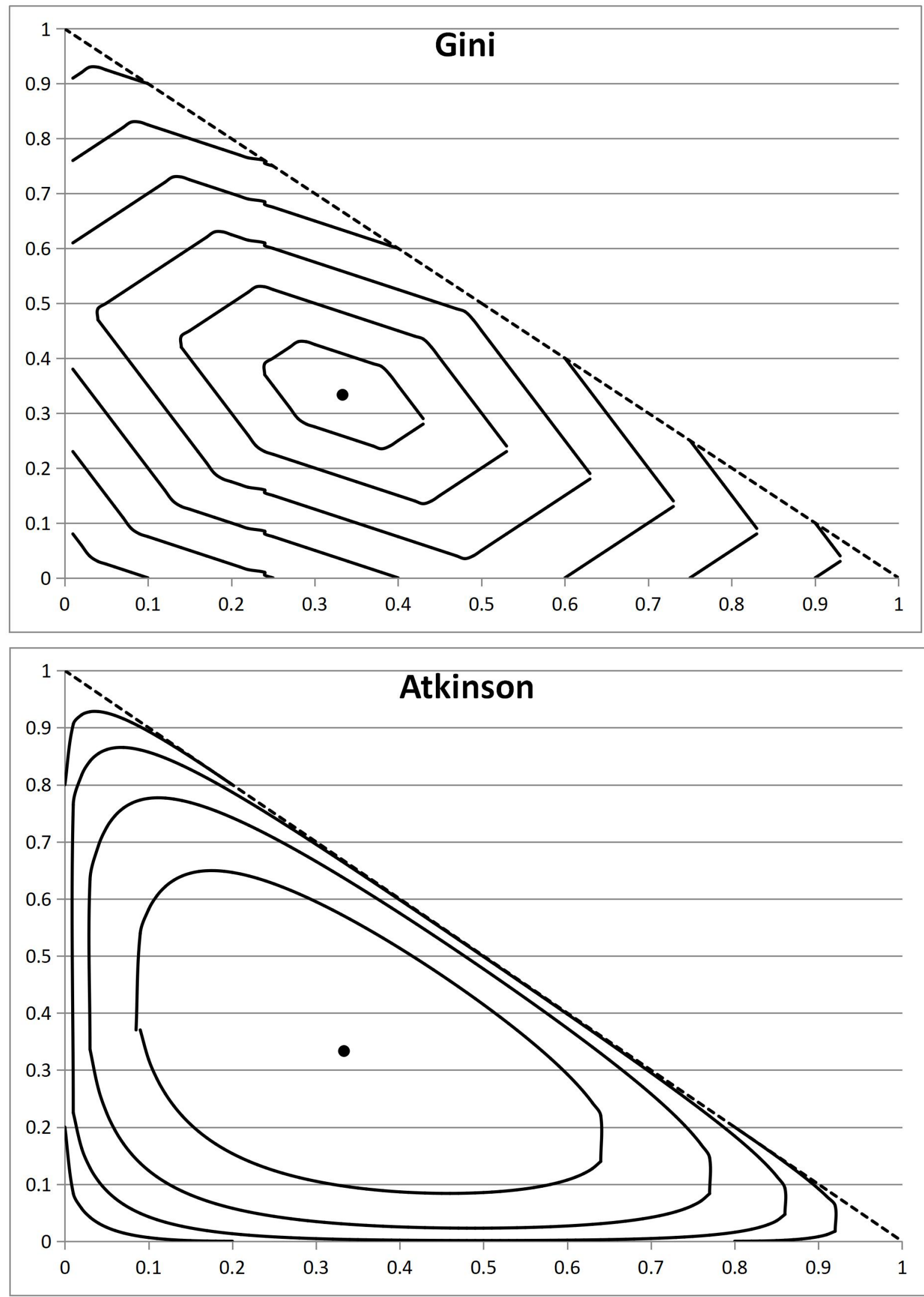

Source: The Author's work 
Figure 3: Contour plots of iso-inequality for chosen measures of inequality, Theil index, and Kolm index with $\alpha=0.5$
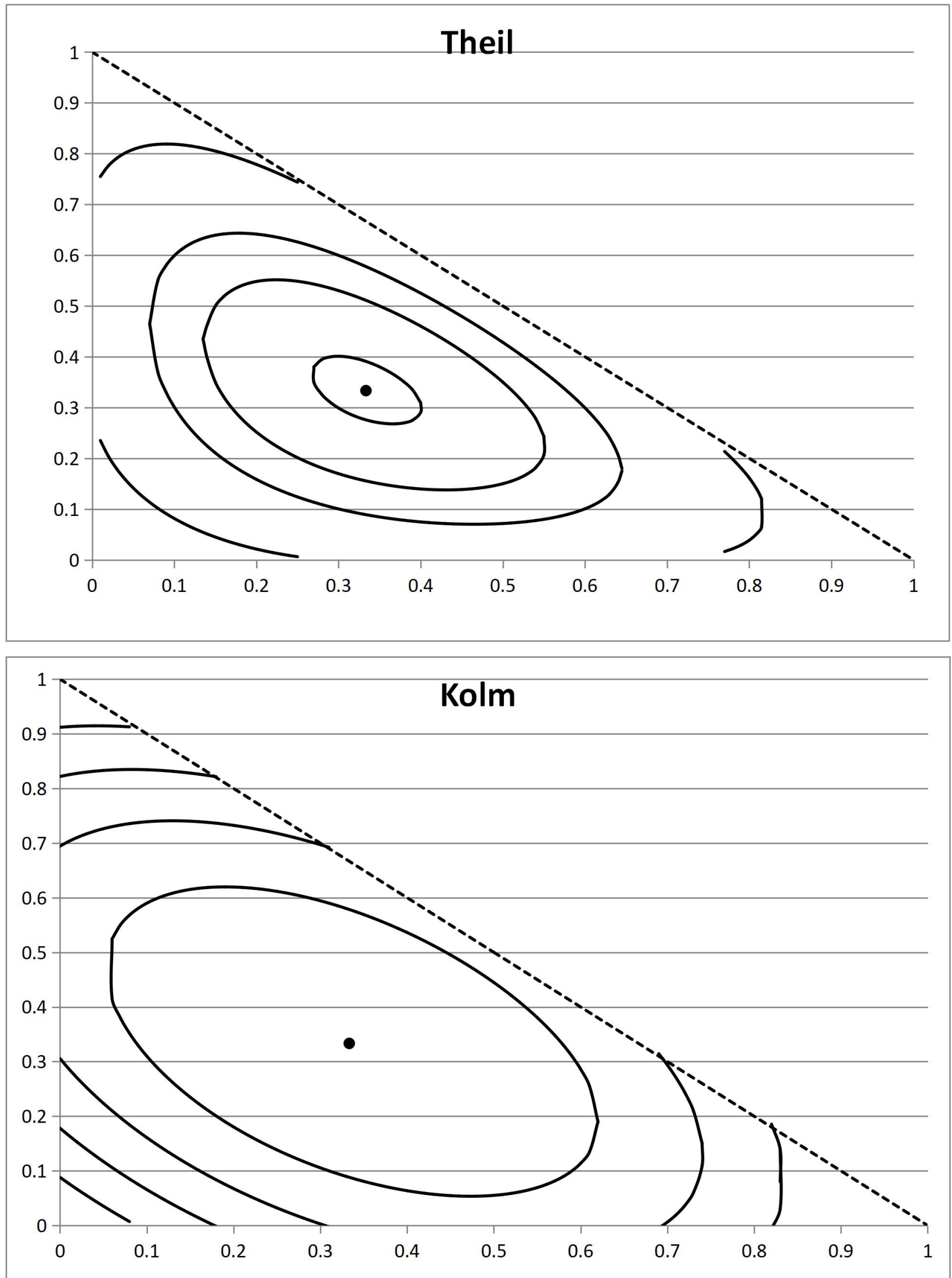

Source: The Author's work 
It may be also worth to invert the perspective and apart from investigate the invariance properties of the measures rather to investigate how they change under the transformations with respect to which they are not invariant. At a first glance, comparing plots for e.g. Atkinson and Kolm measures and not noticing significant differences one may expect to observe something similar for the measure joining properties of the former and the latter.

Figure 4: Contour plots of iso-inequality for $\mathrm{I}_{\mathrm{RL}}, \mathrm{k}=2$

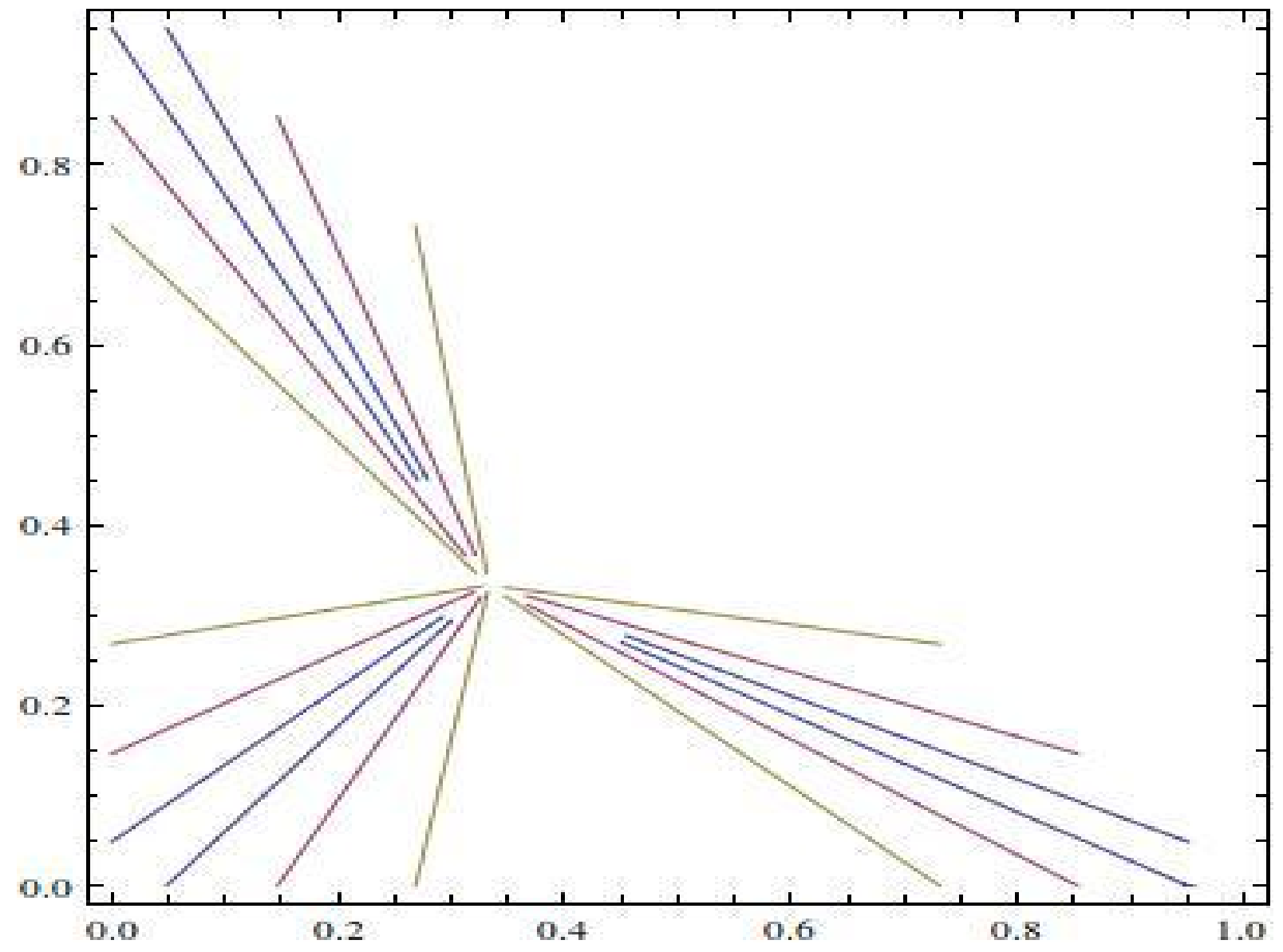

Source: The Author's work

However, this simple view change while we compare how behave these measures with respect to rescaling (Kolm measure, not invariant to such transformation) and translating (Atkinson index, not invariant to such transformation) - see Figure 7 below. If we want to proceed from $T=1$ to $T=2$ - that is, from total resource to be divided from 1 unit to 2 unit - it may be obtained by either multiplying each state of the possession (of three individuals) by the factor 2 or by adding to the state of the possession of each individual $1 / 3$. Multiplying by 2 each value does not change the value of rightist measures, thus, plotting $I_{R}(x, y, 1-x-y)$ and $I_{R}(2 x, 2 y, 2-2 x-2 y)=I_{R}(2(x, y, 1-x-y))$ results in exactly the same surface. Similarly, plotting $I_{L}(x, y, 1-x-y)$ and $I_{L}\left(x+\frac{1}{3}, y+\frac{1}{3}, \frac{4}{3}-x-y\right)=$ $I_{L}\left((x, y, 1-x-y)+\left(\frac{1}{3}, \frac{1}{3}, \frac{1}{3}\right)\right)$ give the same surface. Thus, any interesting effect may appear only if we interchange the measures and transformations, that is, plot $I_{L}(2(x, y, 1-$ $x-y))$ and $I_{R}\left((x, y, 1-x-y)+\left(\frac{1}{3}, \frac{1}{3}, \frac{1}{3}\right)\right)$. 
Figure 5: Equi-inequality contours for $\mathrm{T}=1$ and $\mathrm{T}=2$ for Atkinson index with $\epsilon=0.5$ and Kolm index with $\alpha=0.5$
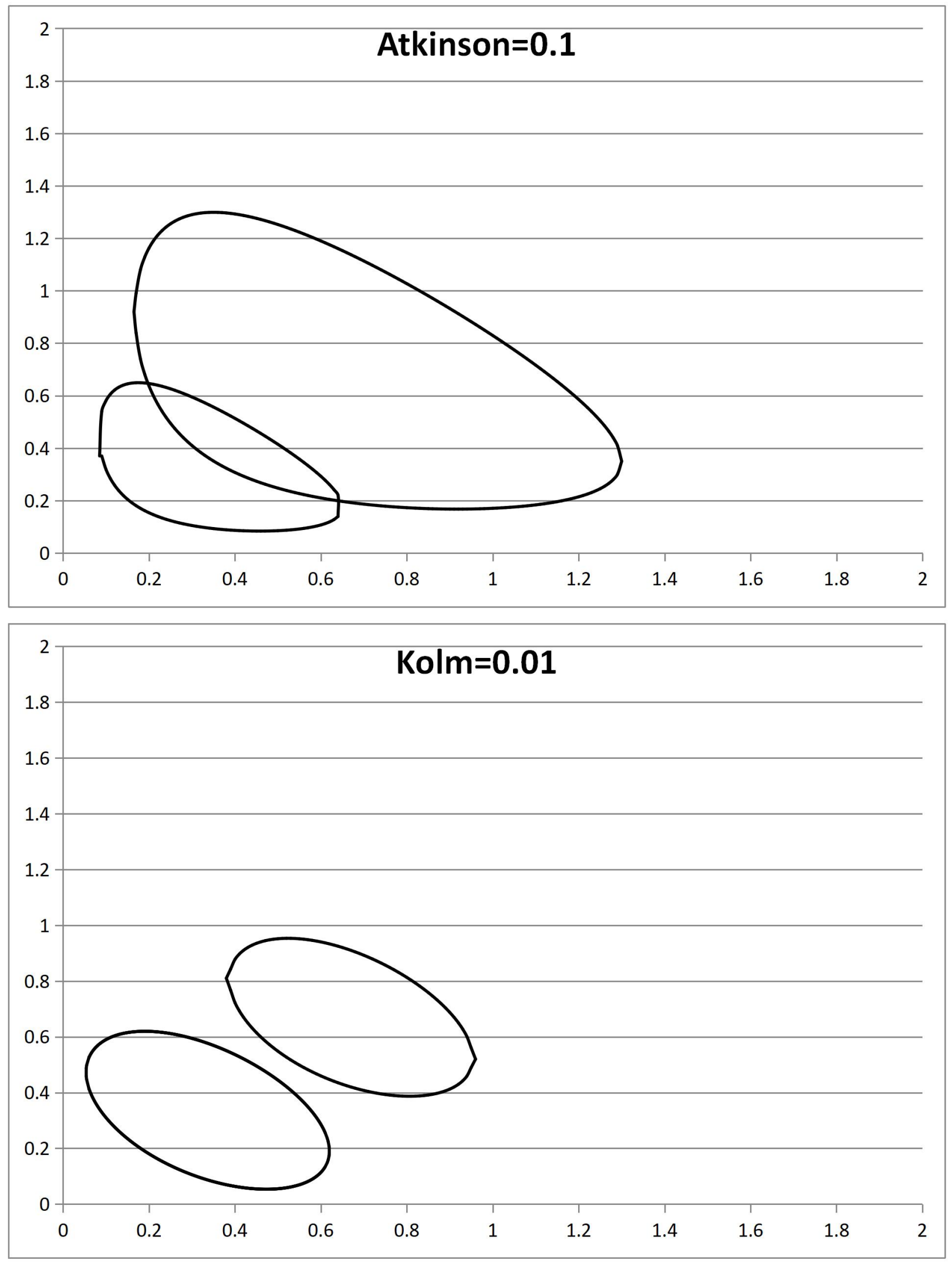

Source: The Author's work 
Figure 6: The shape of $\mathrm{I}(\mathrm{x}, \mathrm{y}, 1-\mathrm{x}-\mathrm{y})$ for Gini index, Atkinson index with $\epsilon=0.5$, Theil index, Kolm index with $\alpha=0.5$ and $\mathrm{I}_{\mathrm{RL}}$ with $\mathrm{k}=2$
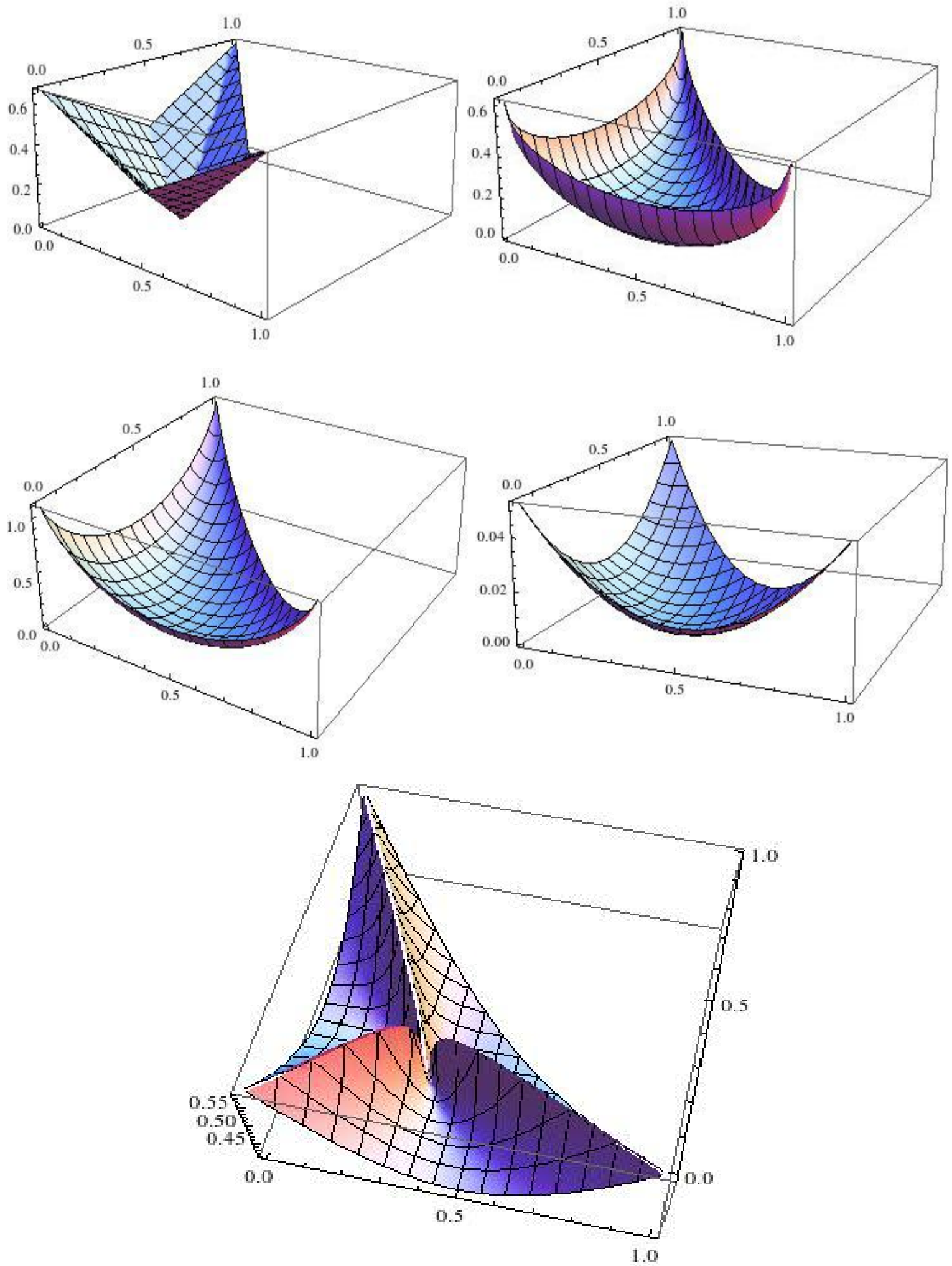

Source: The Author's work

Examining these changes that undergoes rightist measures under translation and leftist under scaling, we have to come to the conclusion that avoiding such effects (as the measure 
having together rightist and leftist properties cannot reveal such changes) has to require very specific shape of $I_{R L}$.

Figure 7: Atkinson index $\left(\mathrm{A}_{0.5}(\mathrm{x}, \mathrm{y}, 1-\mathrm{x}-\mathrm{y})\right.$ - upper part, $\mathrm{A}_{0.5}\left(\mathrm{x}+\frac{1}{3}, \mathrm{y}+\frac{1}{3}, \frac{4}{3}-\mathrm{x}-\mathrm{y}\right)$ lower part) and Kolm measure $\left(\mathrm{K}_{0.5}(\mathrm{x}, \mathrm{y}, 1-\mathrm{x}-\mathrm{y})-\right.$ lower part, $\mathrm{K}_{0.5}(2 \mathrm{x}, 2 \mathrm{y}, 2-2 \mathrm{x}-2 \mathrm{y})-$ upper part
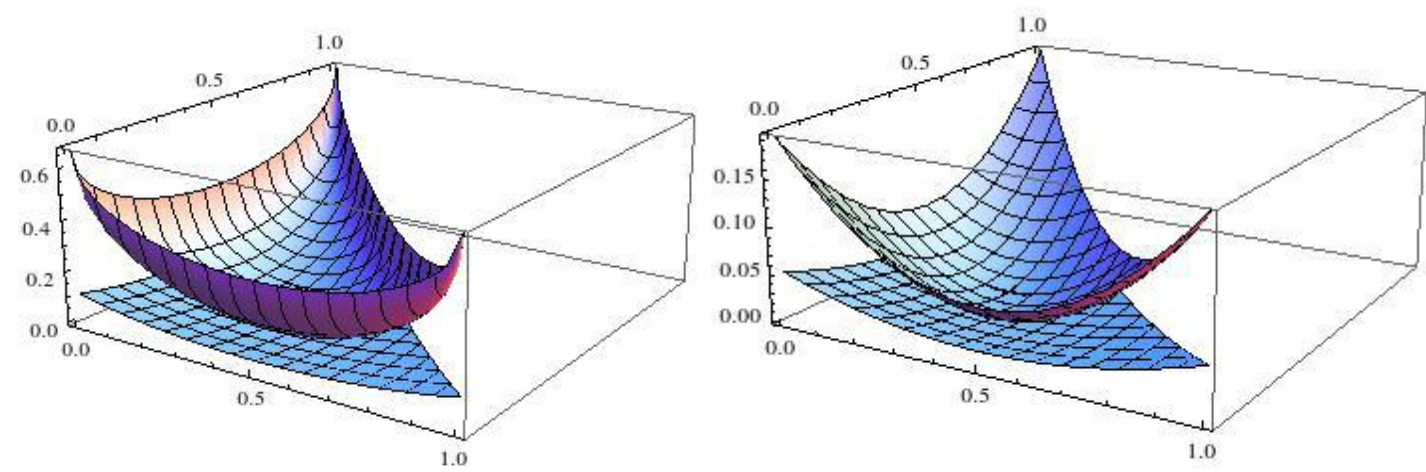

Source: The Author's work

\section{Concluding Remarks}

These are rightist measures which are commonly used both in science and in public communications. The fact is, that using them is much more simple and unproblematic, while proceeding to the leftist measures rises problems with units. However, these problems are not unsolvable, as there is a well-known practice of using e.g. purchasing power for comparisons between different units and different circumstances. Mathematical properties of leftist measures are not yet deeply recognized; still more abandoned are mutually leftist and rightist measures.

Examining the properties of measures that are rightist and leftist at the same time, has the twofold merits. First, due to different attitudes of persons concerning what should be regarded as more/less unequal, these measures might appear to be most compelling with the subjective feelings of at least some sub-group of the population.

Second, as it was shown in the proceeding paragraphs, examining such measures may give an interesting insight into the nature of rightist and leftist measures and deeper recognize similarities and differences between them.

The specific character of the mutually rightist and leftist measure seems to be very promising for further investigations.

\section{References}

[1] Allison, P.D., 1978. Measures of inequality. American sociological review, vol. 43, iss. 6, pp. 865-880.

[2] Amiel, Y., Cowell, F.A., 1999. Income transformation and income inequality. In: Advances in Econometrics, Income Distribution and Scientific Methodology, pp. 209-232. Physica-Verlag HD.

[3] Atkinson, A.B., 1970. On the measurement of inequality. Journal of economic theory, vol. 2 , iss. 3, pp. 244-263. 
[4] Cowell, F., 1985. A fair suck of the sauce bottle'or what do you mean by inequality?. Economic record, vol. 61, iss. 2, pp. 567-579.

[5] Cowell, F., 2011. Measuring inequality. Oxford University Press.

[6] Gehrig, W., 1980. Zur Messung der Ungleichkeit in der Wirtschaft, Karlsruhe.

[7] Gini, C., 1921. Measurement of inequality of incomes. The Economic Journal, vol. 31, iss. 121, pp. 124-126.

[8] Greselin, F., Pasquazzi, L., Zitikis, R., 2010. Zenga's new index of economic inequality, its estimation, and an analysis of incomes in Italy. Journal of Probability and Statistics, vol. 2010, Article ID 718905, pp. 1-26.

[9] Kelley, J., Klein, H.S., 1977. Revolution and the rebirth of inequality: A theory of stratification in postrevolutionary society. American Journal of Sociology, vol. 83, iss. 1, pp. 78-99.

[10]Kjellsson, G., Gerdtham, U.G., 2013. Lost in translation: rethinking the inequality equivalence criteria for bounded health variables. In: Health and Inequality, pp. 3-32. Emerald Group Publishing Limited.

[11] Kolm, S.C., 1976a. Unequal inequalities. I. Journal of Economic Theory, vol. 12, iss. 3, pp. 416-442.

[12] Kolm, S.C., 1976b. Unequal inequalities. II. journal of Economic Theory, vol. 13, iss. 1, pp. 82-111.

[13]Kolm, S.C. 1999. Rational foundations of income inequality measurement. In: Handbook of Income Inequality Measurement, pp. 19-94, Kluwer Academic Publishers.

[14] Kot S.M., 2012, Ku stochastycznemu paradygmatowi ekonomii dobrobytu. Kraków: impuls.

[15] Ostasiewicz, K., 2014. Impact of outliers on inequality measures - a comparison between Polish voivodeships. Silesian Statistical Review vol. 12, pp.105-120.

[16] Theil, H.. 1967. Economics and information theory. Amsterdam: North-Holland. 